\title{
Stair-climbing gait for a four-wheeled vehicle
}

\author{
Shuro Nakajima*
}

\begin{abstract}
This paper proposes a stair-climbing gait for a four-wheeled vehicle. The idea and methodology of climbing stairs are described, and the results of experiments are shown. Crawler-type vehicles and vehicles with special, complicated mechanisms are commonly used for climbing continuous steps (stairs). In contrast, in this paper, a four-wheeled vehicle with additional degrees of freedom, whose mechanism is not as complicated, is discussed. A gait algorithm based on a pace gait is proposed, and its performance is evaluated.
\end{abstract}

Keywords: Climbing stairs, Powered wheelchair, Four-wheeled vehicle, Personal mobility vehicle

\section{Introduction}

It is said that the number of wheelchair users is $1 \%$ of the world population, that is, 77 million people. The growing elderly population has led to increased demand for personal mobility vehicles (PMVs), including powered wheelchairs $[1,2]$.

There are many types of powered wheelchair products; however, their ability to move on steps, stairs and other rough terrain is insufficient for daily activities. Therefore, extensive research and development have been conducted at the university level (prototype level) and by companies (product level).

One of the main barriers for PMVs in terms of rough terrain is stairs. To overcome this barrier, a crawler mechanism is often used at the prototype level. A robotic wheelchair with a wheel-track design [3] uses a track system, not wheels, when climbing up or down stairs. Wheelchair.q [4] is another type of wheelchair for climbing stairs, which has a crawler mechanism.

Special mechanisms other than crawler mechanisms have been developed for climbing stairs. The active rotary-leg mechanism is proposed for climbing stairs in [5]. This mechanism is good for stairs when approaching at a right angle but has not yet been discussed with

*Correspondence: nakajima@wakayama-u.ac.jp

Faculty of Systems Engineering, Wakayama University, 930 Sakaedani, Wakayama-city 640-8510, Japan respect to approaching from different angles. A design for a rough terrain vehicle with a rocker-bogie suspension is discussed in [6]. This mechanism exhibits high capability when moving on rough terrain, but the methodology of actively controlling joints for climbing stairs is not sufficiently addressed. A legged mechanism is proposed for climbing stairs in [7]. The legged mechanism exhibits a high capability for moving on rough terrain, but its mechanism tends to become complicated and exhibits reduced performance on paved terrains.

On the other hand, most commercial wheelchair products for rough terrain are normally a wheeled type [8], with a few examples of the crawler type. The reason for this is based on the following:

- Wheeled-type vehicles can move around faster than crawler-type vehicles.

- Wheeled-type vehicles require less energy to move around than crawler-type vehicles.

There are studies that modify and improve conventional vehicles. An active-caster add-on mechanism to enable a manual wheelchair to climb a step is proposed in [9]. This type of add-on mechanism is useful for manual wheelchairs, but its performance for stairs is limited. MEBot [10] has been developed to enhance daily activity for people with disabilities, and numerous studies have been 
conducted from the perspective of users. MEBot can move over a step but cannot climb stairs.

Overall, there is not yet a good solution for a powered wheelchair with sufficient mobility performance in addition to high speed and high energy efficiency. Therefore, we have been developing and studying the RT-Mover series $[11,12]$. The concepts behind this series are as follows:

- The wheel mechanism is primarily used to achieve high speed capability and high energy efficiency when moving on paved roads, which are the main environment for a PMV.

- The wheel mechanism can perform a leg-like motion if necessary.

- The driver's seat posture is controlled to remain horizontal even when moving on rough terrain.

- For the sake of simplicity, the number of actuators is reduced to the minimum necessitated by the motion being performed.

The RT-Mover series is one type of leg-wheel robot. Studies on leg-wheel robots have been conducted, and the leg-wheel hybrid quadruped robot [13] is one good example. In [13], a static friction condition for a wheel mechanism climbing steps is formulated. The leg-wheel hybrid quadruped robot [13] has the minimum number of active shafts, assuming that the wheels are always in contact with the terrain. While the RT-Mover series are different from this robot regarding the number of active shafts and the existence of a body part that can be maintained in a horizontal position, we refer to the research [13].

\section{Previously proposed leg motion and its limitations}

Figure 1 shows the RT-Mover PType WA Mk-II (P-WA Mk-II), which is the target vehicle of this paper. There are four active wheels. A steering axis and a rolling axis are equipped at the center of both the front and rear wheel arms. The driver's seat slides left/right and forward/ backward with pitching. A robotic arm is installed on the right armrest but is not considered in this paper. Table 1 shows the main specifications of the P-WA Mk-II.

The performance of the leg motion of the P-WA is discussed in [14]. In the proposed leg motion, one wheel is lifted to perform the leg motion, while the other three wheels support the body (Fig. 2). When the center of gravity of the vehicle is within the support polygon, the leg-motion wheel can be lifted like a leg. During the legmotion wheel's forward movement from (a) of Fig. 2 to (b), the support wheels must be moved according to the motion of the leg-motion wheel. Assuming that the situation of the P-WA is that in Fig. 3 or the upper left image

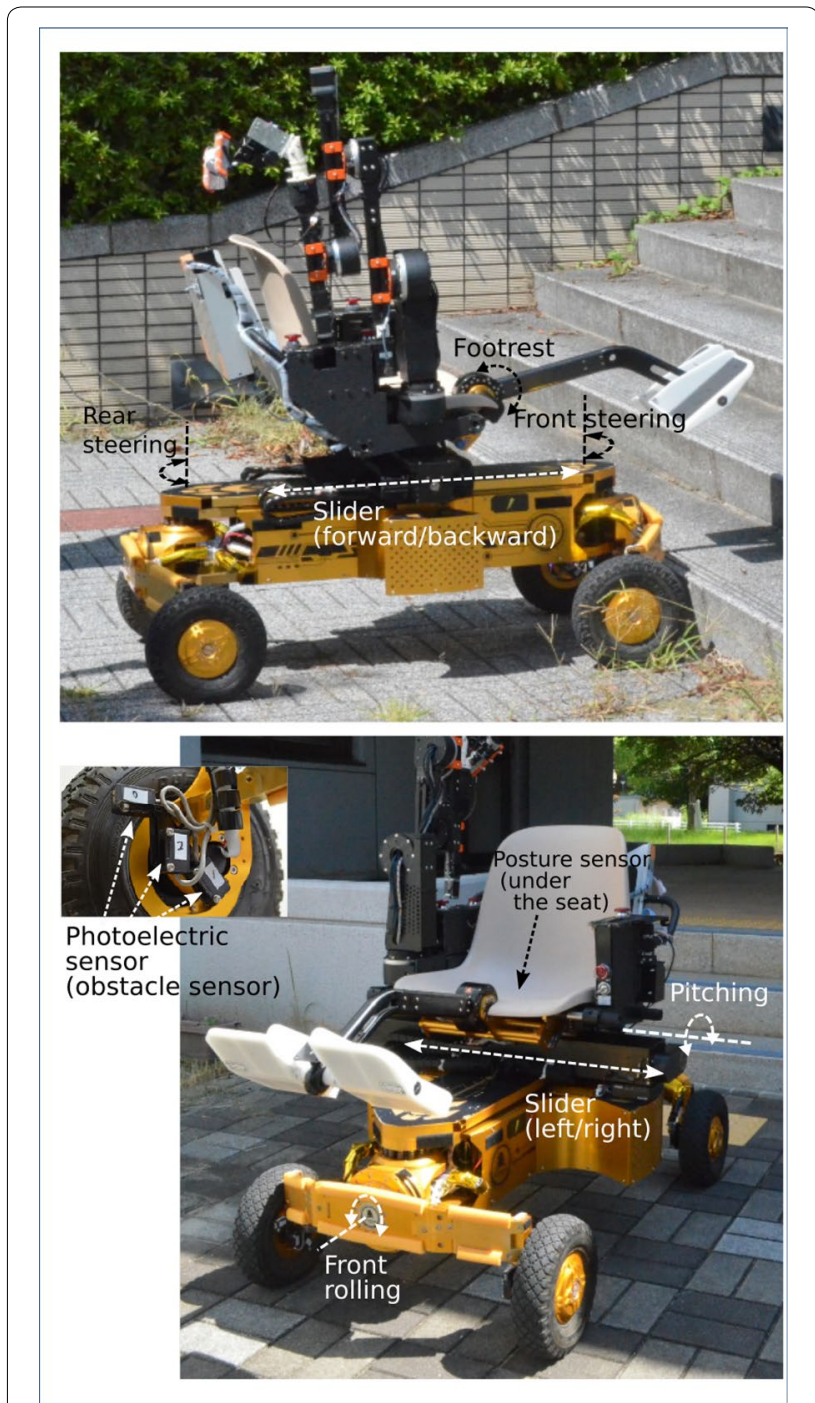

Fig. 1 RT-Mover PType WA Mk-II

(2 [s]) of Fig. 10, even if any wheel can be selected as the leg-motion wheel, the support wheels must contact the step. Since the support wheels do not have the capability of going over a step, the leg motion cannot be completed, and the P-WA cannot climb the step in the case of Fig. 3 or the upper left image of Fig. 10. The leg-wheel hybrid quadruped robot [13] also cannot complete the movement in the same situation, since it is difficult for multiple wheels to climb steps simultaneously.

In this paper, we propose a methodology to address this problem with the following policies:

- The static friction condition for a wheel mechanism climbing a step was analyzed in [13], and we use that condition here. 
Table 1 Main specifications of the P-WA MK-II

\begin{tabular}{|c|c|}
\hline Body dimensions & $\begin{array}{l}\text { Length: } 1.1 \mathrm{~m} \text {; width: } 0.7 \mathrm{~m} \text { (not including the } \\
\text { footrest) }\end{array}$ \\
\hline Wheel & Radius: 0.125 m; width: $0.07 \mathrm{~m}$ \\
\hline Weight & $92 \mathrm{~kg}$ (including the battery) \\
\hline Motor & $\begin{array}{l}200 \mathrm{~W} \text { (wheels); } 150 \mathrm{~W} \text { (front/rear rolling); } 100 \mathrm{~W} \\
\text { (others) }\end{array}$ \\
\hline Gear ratio & $\begin{array}{l}100 \text { (wheels); } 960 \text { (front/rear rolling); } 3855 \text { (pitch- } \\
\text { ing); } 2800 \text { (front/rear steering); } 15+10 / 30[\mathrm{~mm} \text { - } \\
\text { lead ball screw (lateral/longitudinal slider) }\end{array}$ \\
\hline Main sensors & $\begin{array}{l}\text { Posture angle sensor (roll and pitch of the seat); } \\
\text { photoelectric sensor (12,3 per wheel) }\end{array}$ \\
\hline Angle limit & $\pm 27^{\circ}$ (pitch axis); $\pm 35^{\circ}$ (steering and roll axis) \\
\hline Slider limit & $\begin{array}{l} \pm 0.3 \mathrm{~m} \text { (left/right); } 0.45 \text { to }-0.15 \mathrm{~m} \text { (forward/ } \\
\text { backward) }\end{array}$ \\
\hline Max speed & $7.4 \mathrm{~km} / \mathrm{h}$ (catalog value) \\
\hline Power supply & $48 \mathrm{~V}$ (lithium polymer battery) \\
\hline
\end{tabular}

- The proposed methodology is not a method of solving target values analytically but a set of rules gov-

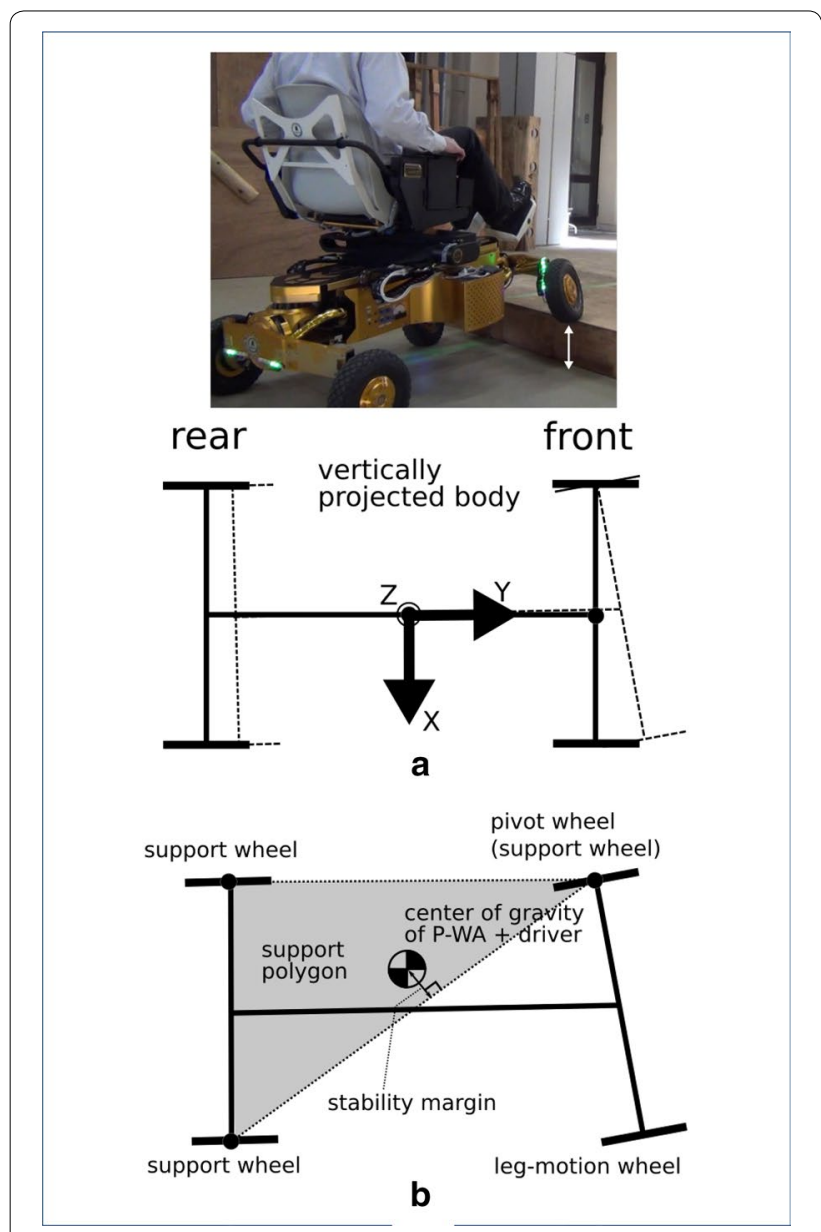

Fig. 2 Leg motion for the P-WA

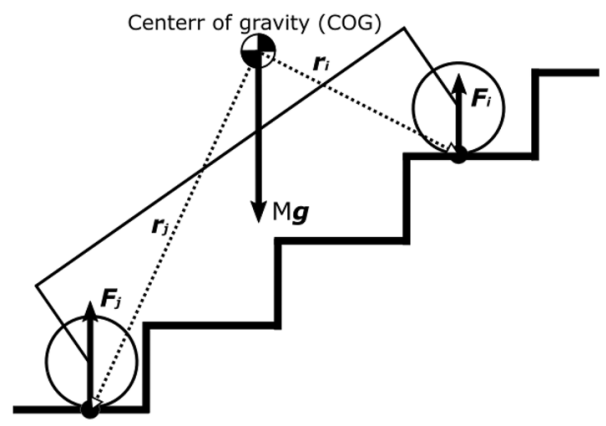

Fig. 3 Vehicle on a set of stairs

erning the motion and control of our vehicle, since, for example, load distribution across four wheels occurs in statically indeterminate conditions.

\section{Gait for climbing stairs}

A gait by which the P-WA Mk-II can climb stairs is proposed in this section. The idea of the stair-climbing gait is as follows:

- The four wheels are assumed to follow a terrain surface.

- The four wheels are divided into two categories: pivot wheels and moving wheels.

- A moving wheel has the capability of going over a step when facing the step.

The stair-climbing gait is considered a static physical phenomenon, and we assume that the times at which a front wheel and a rear wheel face a step during the pace gait as stated below are not exactly the same.

In statics, the basic formulas in Fig. 3 are as follows:

$$
\begin{aligned}
& \sum_{i=1}^{4} \boldsymbol{F}_{\boldsymbol{i}}=M \boldsymbol{g}, \\
& \sum_{i=1}^{4}\left(\boldsymbol{r}_{\boldsymbol{i}} \times \boldsymbol{F}_{\boldsymbol{i}}\right)=0,
\end{aligned}
$$

where $\boldsymbol{F}_{\boldsymbol{i}}$ is the reaction force from the terrain, $\boldsymbol{r}_{\boldsymbol{i}}$ is the vector from the center of gravity (COG) to the reaction force, $i$ is the leg number, $M$ is the mass of the vehicle, and $g$ is the acceleration due to gravity. The vehicle does not fall down when all $\boldsymbol{F}_{\boldsymbol{i}} \geq 0$. The problem is that it is difficult to determine the value of each $\boldsymbol{F}_{\boldsymbol{i}}$ due to indeterminate conditions. Therefore, we set rules governing the motion and control of our vehicle, and consequently, the values in indeterminate conditions are decided. 


\section{Numbers of pivot wheels and moving wheels}

Regarding the mechanism of the P-WA Mk-II, there is no room for three pivot wheels and one moving wheel. On the other hand, with one pivot wheel and three moving wheels, the loads of the three wheels should decrease in order for each moving wheel to climb a step. In other words, the weight should be one-sided with respect to the single pivot wheel, but this effect is constrained. Therefore, a configuration with two pivot wheels and two moving wheels is the best choice for the proposed gait.

\section{Suitable gait for climbing stairs}

There are three gait patterns for a four-legged animal that has two pivot legs for walking or running: a trot gait, a pace gait and a gallop gait. Due to the mechanism of the P-WA Mk-II, the trot and pace gaits can be achieved. Regarding the trot gait (Fig. 4 left), a moving wheel exists at each side of the body (left/right and front/rear). This means that there is no position of the P-WA Mk-II with a one-sided center of gravity (COG), because one moving wheel is positioned at each side of the body. In the case of the pace gait (Fig. 4 right), a pivot wheel exists on one side (left/right). Therefore, it is possible for the COG to be one-sided with respect to the pivot side by moving the upper part of the P-WA Mk-II, including the driver. Therefore, the pace gait is chosen as the proposed gait.

\section{Condition for a wheel to climb a step}

Figure 5 shows the analysis for a moving wheel climbing a step. Force $\boldsymbol{F}_{\boldsymbol{c}}$ is the load on the wheel. Force $\boldsymbol{F}_{\boldsymbol{b}}$ is the upward force generated by the actuator of the rolling axis. Force $\boldsymbol{F}_{\boldsymbol{a}}$ is the driving force of a wheel (friction force between the wheel and the front surface of the step). Force $\boldsymbol{F}_{\boldsymbol{a}}$ is determined by a pushing force $\boldsymbol{F}_{\boldsymbol{d}}$, which is generated by the actuator of the steering axis. $\left|\boldsymbol{F}_{\boldsymbol{a}}\right|$ is less than $\mu\left|\boldsymbol{F}_{\boldsymbol{d}}\right|$ (where $\mu$ is the coefficient of friction). If the following equation is true, then the wheel can move upward:

$$
\left|\boldsymbol{F}_{\boldsymbol{a}}\right|+\left|\boldsymbol{F}_{\boldsymbol{b}}\right|>\left|\boldsymbol{F}_{\boldsymbol{c}}\right|
$$

For the pace gait, all moving wheels must satisfy Eq. (3).

\section{Center of gravity of the P-WA Mk-II including a driver}

When a moving wheel faces a step, it can climb the step under Eq. (3). To easily satisfy this equation, the COG of the P-WA Mk-II including a driver should be one-sided with respect to the pivot wheel side so that the load of the moving wheels decreases and the maximum value of the pushing force, $\boldsymbol{F}_{\boldsymbol{d}}$ in Fig. 5, is enhanced due to the larger load of the pivot wheels (if the pivot wheel load is small, then the pivot wheels may slip when generating a pushing force). To adjust the COG position, slider mechanisms (left/right and forward/backward) are used. If the COG is positioned within the triangular zone formed by the two pivot wheels and the center of the body shown in Fig. 6, then two pivot wheels and one moving wheel can support the body even when the other moving wheel is lifted off of the ground. Therefore, the COG should always be located within the triangular zone during the proposed gait.

\section{Control methodology of the main joints}

In this section, the control laws or rules for achieving the proposed gait are described.

\section{Rolling axis}

By applying Eq. (4) to the front and rear rolling axes, the driver's seat is maintained in a horizontal position even on rough terrain.

$$
T_{d}=K\left(\theta_{d}-\theta\right)+D\left(\dot{\theta}_{d}-\dot{\theta}\right),
$$

where $T_{d}$ is the target torque, $\theta$ is the seat angle, $\theta_{d}$ is the target seat angle, $K$ is the angle gain, and $D$ is the angular velocity gain.

The target angle $\theta_{d}$ of the front and rear rolling axes is normally 0 , while when a moving wheel is climbing a step, the $\theta_{d}$ of the rolling axis that faces the step is inclined toward the step.

When a moving wheel contacts a step, the moving wheel is lifted over the step, and the rolling angle of the driver's seat is tilted. By controlling Eq. (4), $\boldsymbol{F}_{\boldsymbol{b}}$ in Fig. 5 is then increased to lift the wheel, and the wheel is lifted more. This cycle continues until the top of the step is reached.

The other rolling axis is mainly for maintaining the rolling posture of the seat.

\section{Steering axis and wheel}

The steering axis and wheel are controlled to generate the pace gait.

When a moving wheel faces a step, the steering velocities on both sides are reduced while climbing the step, and the velocity of the moving wheel is increased in going up the step. The moving wheel is pushed by the steering axis, and then the maximum friction force $\boldsymbol{F}_{\boldsymbol{a}}$ in Fig. 5 increases and $\boldsymbol{F}_{\boldsymbol{b}}$ in Fig. 5 also becomes larger, as mentioned above. When the condition of the moving wheel (Eq. (3)) is satisfied, the wheel climbs the step.

\section{Pitching axis}

For the driver's comfort and to adjust the position of the COG, the pitch axis is controlled by Eq. (4). The target angle $\theta_{d}$ of the pitch axis is 0 ; therefore, the driver's seat is kept horizontal within angle limitations. Since the angle 


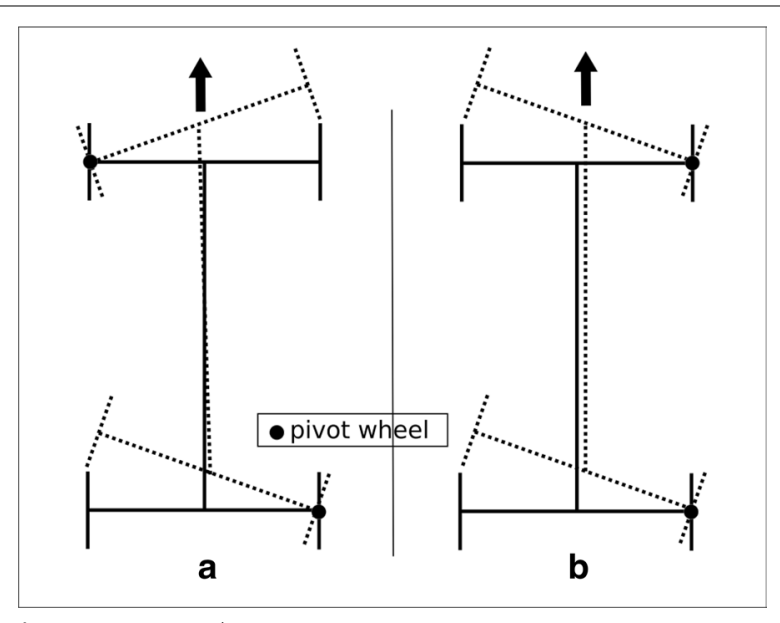

Fig. 4 Trot gait and pace gait

limitation is $\pm 27 \mathrm{deg}$, the seat pitch angle is tilted when this limitation is surpassed.

\section{Experimental setup}

When the COG overall is within the triangular zone of Fig. 6 and the proposed gait is directed according to the control law of Eq. (4), a moving wheel can climb a step if it satisfies the condition of Eq. (3). Table 2 and Fig. 7 show the experimental setup for the proposed motion. These values are determined experimentally in the first step.

When the driver's seat is moved to the position of the seat slider $\mathrm{LR}=0.20 \mathrm{~m}$ and seat slider $\mathrm{FB}=0.26 \mathrm{~m}$ at the beginning of the proposed gait on the stairs, as shown in the image at $3 \mathrm{~s}$ in Fig. 10, the COG of the P-WA Mk-II and a driver weighing $65 \mathrm{~kg}$ is $0.11 \mathrm{~m}$ to the right and $0.036 \mathrm{~m}$ back from the center of the projected frame on the horizontal plane, which is within the triangular zone shown in Fig. 6. This COG position can sufficiently maintain the positive stability margin in Fig. 2 during the proposed gait. When the side of the moving wheels changes from left to right at $9 \mathrm{~s}$ in Fig. 10, the position of the seat slider LR changes from 0.20 to $-0.20 \mathrm{~m}$ to move the COG into the next triangular zone for stability.

The stair-climbing gait begins when the command is given by the driver. After one footstep is finished, the complementary footstep begins if the obstacle sensor at the wheel (Fig. 1) is ON for an obstacle.

When the steering torque is over $50 \mathrm{~N}$ and the obstacle sensor at the wheel is ON for an obstacle, the system detects a step. After detecting the step, the angular velocity of the steering axis reduces to half of the normal value so that the pushing force on the step $\boldsymbol{F}_{\boldsymbol{d}}$ in Fig. 5 is maintained to generate $\boldsymbol{F}_{\boldsymbol{a}}$ in Fig. 5. At the same time, the target seat angle of the rolling axis is changed to $4 \mathrm{deg}$

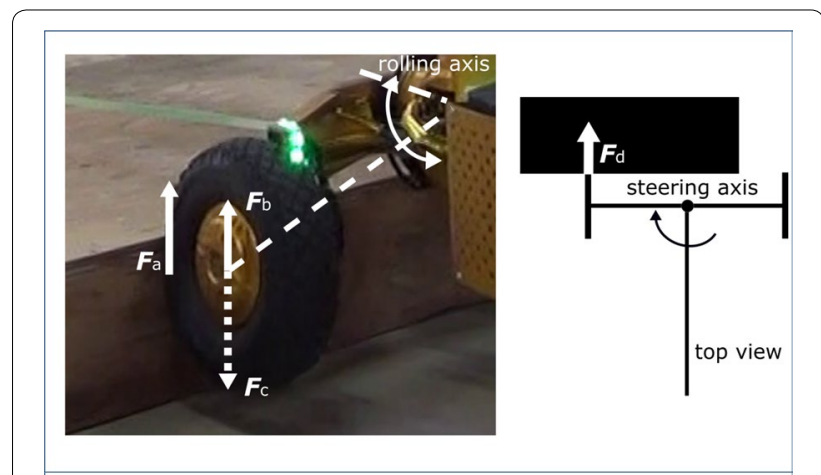

Fig. 5 Force for a wheel to climb a step

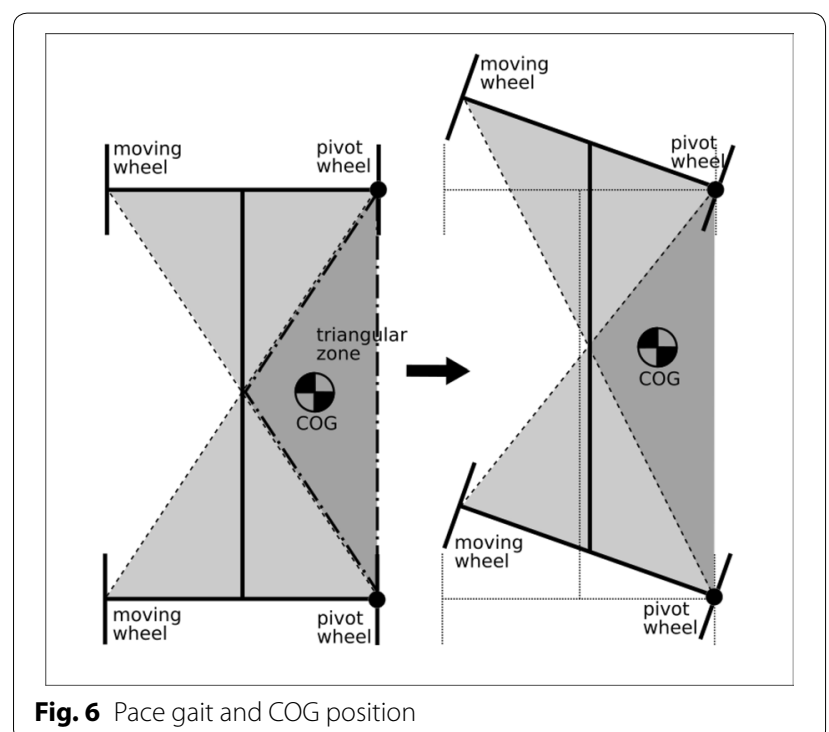

when the moving wheels are on the left side or $-4 \mathrm{deg}$ when they are on the right side, and the wheel velocity is changed from 0.16 to $0.21 \mathrm{~m} / \mathrm{s}$. By changing the target seat angle of the rolling axis, $\boldsymbol{F}_{\boldsymbol{b}}$ in Fig. 5 increases. Once a step is detected, this mode continues up to a rolling axis change of \pm 8 deg to avoid unstable motion.

In this paper, the footstep angle, for example, is constant at 33 deg. If a step exists within the footstep angle and the condition of Eq. (3) is satisfied by using the proposed method as in Eq. (4), the wheel at the step can climb it. On the other hand, there is a size limitation when handling stairs, since some values in Table 2 are constant. These values should be adjusted by measuring the environment to increase the capability of climbing various sizes of stairs; however, as a first step, this paper shows that the proposed gait is possible with the setup values in Table 2 . We will study a method of adjusting values according to an environment, including a driver's weight, by using sensors as a next step. 
Table 2 Experimental setup

\begin{tabular}{ll}
\hline Footstep angle of the steering axis & $33 \mathrm{deg}$ \\
Angular velocity of the steering axis & $14.3 \mathrm{deg} / \mathrm{s}$ \\
Velocity of a moving wheel & $0.16 \mathrm{~m} / \mathrm{s}$ \\
Seat slider LR & $0.20 /-0.20 \mathrm{~m}$ (moving \\
& wheel: left/right) \\
Seat slider FB & $0.26 \mathrm{~m}$ \\
$\theta_{h}$ in Fig. 7 & $35 \mathrm{deg}$ \\
h in Fig. 7 & $0.51 \mathrm{~m}$ \\
$I_{1}$ in Fig. 7 & $0.18 \mathrm{~m}$ \\
$I_{2}$ in Fig. 7 & $0.23 \mathrm{~m}$ \\
$I_{3}$ in Fig. 7 & $0.30 \mathrm{~m}$ \\
Weight of the upper part including the driver & $85 \mathrm{~kg}$ \\
Weight of the lower part & $72 \mathrm{~kg}$ \\
Threshold of the steering torque & $50 \mathrm{~N}$ \\
Target seat rolling axis angle during step & $4 /-4 \mathrm{deg}$ (moving \\
detection & wheel: left/right) \\
Angular velocity of the steering axis during step & $7.2 \mathrm{deg} / \mathrm{s}$ \\
detection & \\
Velocity of a moving wheel during step detection & $0.21 \mathrm{~m} / \mathrm{s}$ \\
$\theta_{1}$ in Fig. 7 (due to exceeding the angle & $8 \mathrm{deg}$ \\
limitation of the pitch axis) & \\
\hline
\end{tabular}

\section{Experimental results}

Figures 8, 9, and 10 show the results of the stair-climbing gait. The Additional file 1 is a film of this experiment. The stair step height is $0.17 \mathrm{~m}$, and the stair step depth is 0.28 $\mathrm{m}$. The driver's weight is $65 \mathrm{~kg}$. The proposed gait was executed without issue, as shown in Fig. 10. The front steering angles in Figs. 8 and 9 are the same. The data of the "lifting FL/RL wheel" in Fig. 8 indicate the timing of lifting the wheel. The actual value is ON (1) or OFF (0);

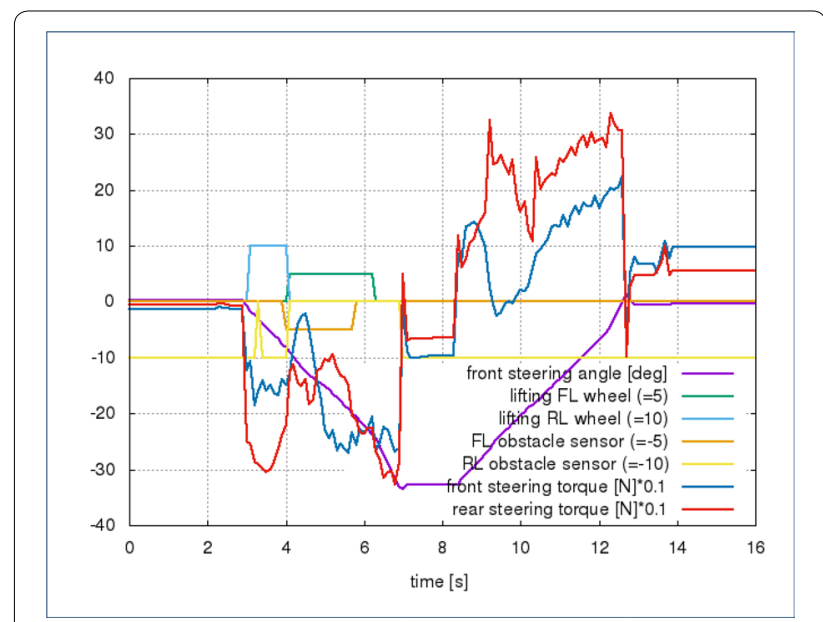

Fig. 8 Data 1 during the stair-climbing gait

however, in the graph, the value is multiplied by 5 or 10 for ease of understanding. Figure 8 shows the flow of detecting a step, and Fig. 9 shows the movement of each angle and the motion of the proposed gait.

When the proposed gait is continuously used, the P-WA Mk-II can climb stairs. Figure 11 and the Additional file 2 show the results of the stair-climbing experiment. The P-WA Mk-II with a driver can climb from the third step to the sixth step of the stairs. In the video, there were some intervals between the stairclimbing gaits because the driver gave the command to start after carefully checking the situation.

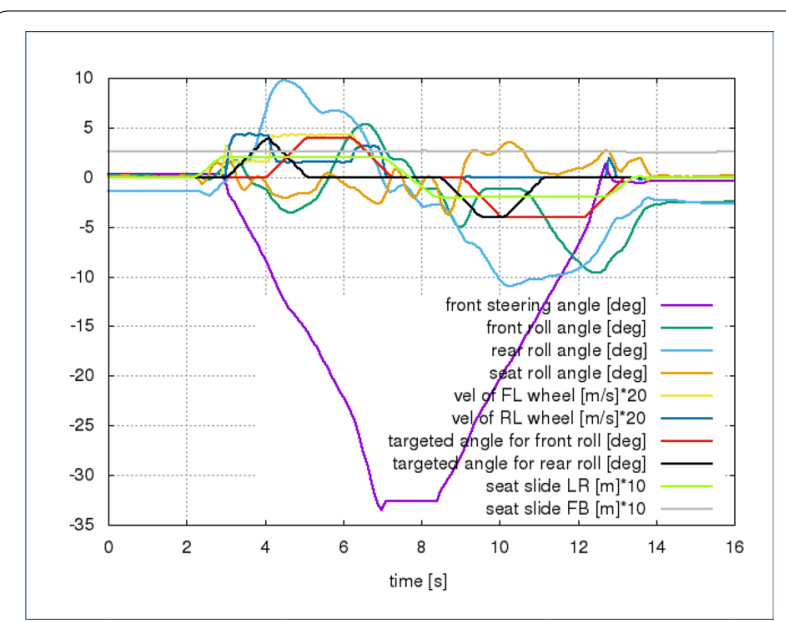

Fig. 9 Data 2 during the stair-climbing gait

Fig. 7 Experimental setup

(b) Top view

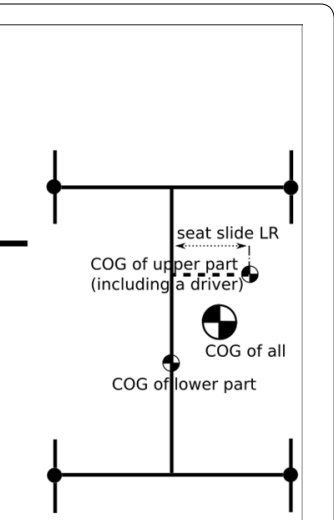

(b) Top view 

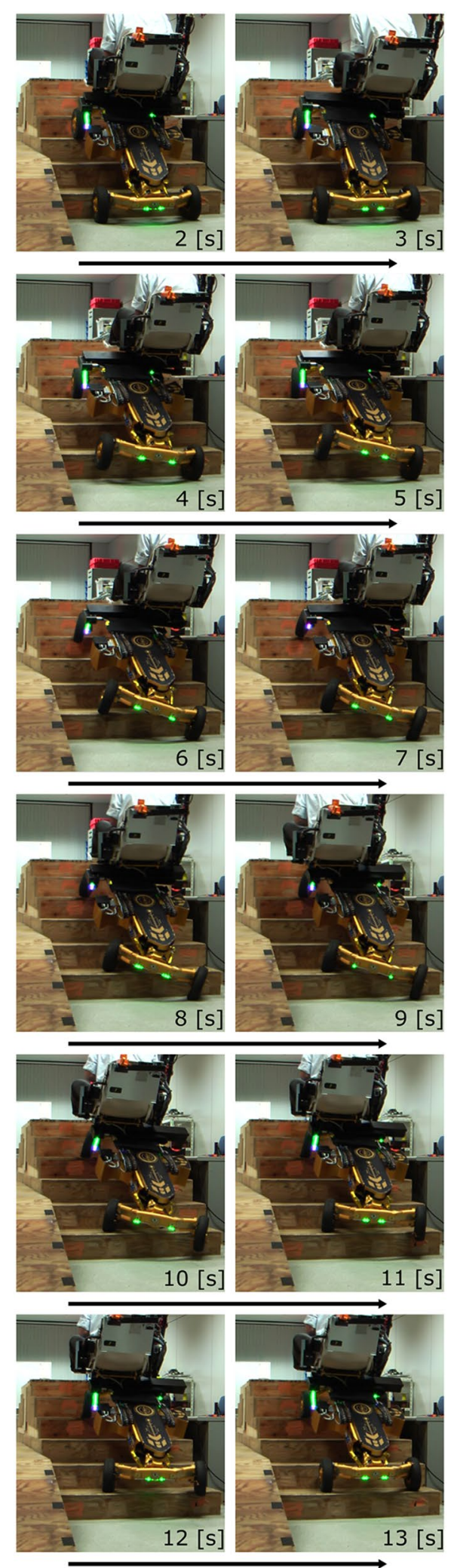

Fig. 10 Stair-climbing gait
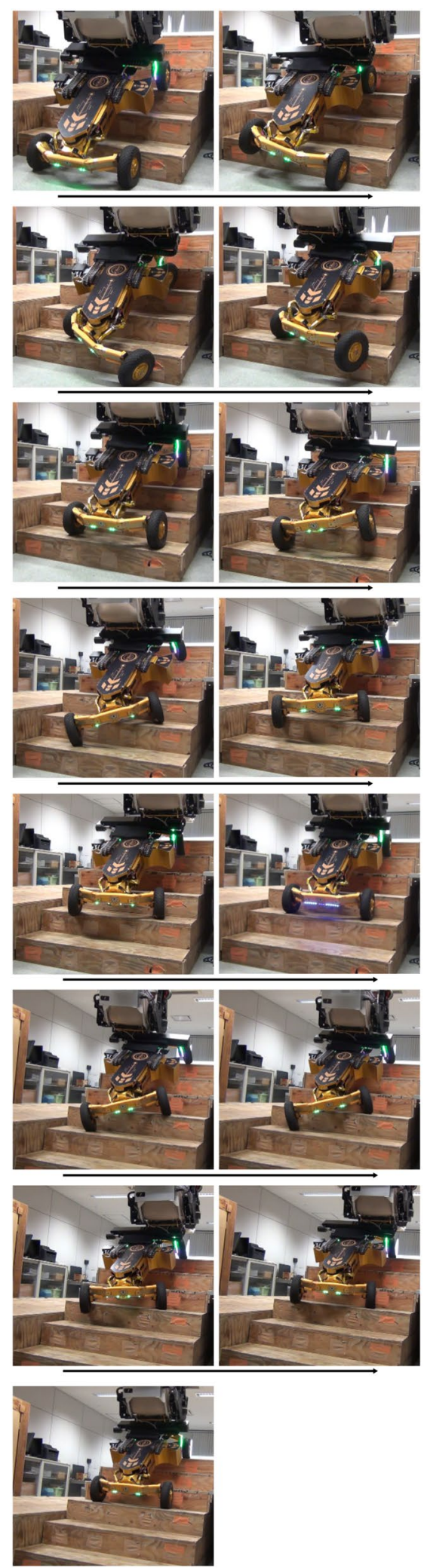

Fig. 11 Climbing up stairs 


\section{Conclusion}

In this paper, we propose a stair-climbing gait for a four-wheeled vehicle. The proposed gait is a pace gait with leveling control for the driver's seat. The position of the COG is adjusted, and actuators are controlled so that a moving wheel can climb a step. By using the proposed gait, it is confirmed that the P-WA Mk-II with a driver can climb up stairs.

The next step is to analyze the limitations of the proposed gait, to improve performance with respect to speed and force distribution and to develop a way to adjust the control values by using environmental data.

\section{Supplementary information}

Supplementary information accompanies this paper at https://doi. org/10.1186/s40648-020-00168-z.

Additional file 1. The video clip of the proposed stair-climbing gate for Figs. 8, 9 and 10 .

Additional file 2. The video clip for the vehicle to climb a stairs for Fig. 11

\section{Acknowledgements}

The experiments were supported by students in our laboratory.

\section{Authors' contributions}

SN proposed the stair-climbing gait for the four-wheeled vehicle, conducted the experiments, and analyzed the data. SN is fully responsible of this paper. All authors read and approved the final manuscript.

\section{Funding}

This work was partially supported by JSPS KAKENHI (Fund for the Promotion of Joint International Research, Grant Number 17KK0006).

\section{Availability of data and materials}

The datasets saved and analysed during the current study are available from the corresponding author on reasonable request. And, movie files generated during this study are included in this published article's Additional files: Additional file 1 for Figs. 8, 9, and 10, Additional file 2 for Fig. 11.

\section{Competing interests}

The authors declare that they have no competing interests.

\section{Author's information}

Shuro Nakajima is currently a Professor at the Faculty of Systems Engineering, Wakayama University, Japan. He received the M.S. and Ph.D. degrees in information science from Tohoku University, Japan. He finished his Ph.D. work in two years. He was enrolled with the Graduate School, Tohoku University, in 1995, by grade skipping. He has been licensed to a Professional Engineer, Japan and registered as a APEC Engineer and an International Professional Engineer, Japan. He was with East Japan Railway Company from 1997 to 2003 and with the Department of Advanced Robotics, Chiba Institute of Technology, from 2005 to 2015. He was also a Visiting Scholar at University of California, Berkeley, from 2013 to 2014. He is a Guest Professor at ETH Zurich in Switzerland from 2020 to 2021. His research interests include personal mobility vehicles, mobile robots, intelligent vehicles, and assistive technology. He was a recipient of the German Innovation Award 2017, IEEJ Technical
Development Award 2017, JSME Kansai Academic Award 2018, the 2012 Best Paper Award of the Japan Society for Design Engineering and more. RT-Mover P-WA, his innovation, received the fourth place award in the powered wheelchair discipline at Cybathlon in 2016.

Received: 9 December 2019 Accepted: 2 April 2020

Published online: 18 April 2020

\section{References}

1. Simpson RC (2005) Smart wheelchairs: a literature review. J Rehabil Res Dev 42:423-436

2. Simpson RC, LoPresti EF, Cooper RA (2008) How many people would benefit from a smart wheelchair? J Rehabil Res Dev 45(1):53-71

3. Podobnik J, Rejc J, Sebastjan S, Marko M, Matjaž M (2017) All-terrain wheelchair. IEEE Robot Autom Mag 24(4):26-36

4. Quaglia G, Nisi M (2017) Design of a self-leveling cam mechanism for a stair climbing wheelchari. Mech Mach Theory 112:84-104

5. Sasaki K, Suzuki KS (2018) Active rotary-legs mechanism for stair-climbing mobility vehicle. IEEE Robot Autom Lett 3(3):2237-2244

6. Dzieżek K, et al (2018) Design and static tests of an all-terrain suspension system for electric wheelchair. In: Proceedings of 12th international conference biomdlore. https://doi.org/10.1109/BIOMDLORE.2018.8467186

7. Pravat KB, Ankur G (2018) Novel design of stair climbing wheelchair. J Mech Sci Technol 32(10):4903-4908

8. EXTREME X8 web site. https://mobility-usa.com/p-extreme-x8.php. Accessed 1 Feb 2020

9. Munakata Y, Wada M (2015) A novel step climbing strategy for a wheelchair with active-caster add-on mechanism. In: Proceedings of 2015 IEEE/RSJ international conference on intelligent and systems, pp 6324-6329

10. Candiotti JL, Kamaraj DC, Daveler B, Cheng-Shiu C, Garrett GG, Rosemarie C, Rory AC (2019) Usability evaluation of a novel robotic power wheelchair for indoor and outdoor navigation. Arch Phys Med Rehabil 100(4):627-637

11. Nakajima S (2011) RT-Mover: a rough terrain mobile robot with a simple leg-wheel hybrid mechanism. Int J Robot Res 30(13):1609-1626

12. Nakajima S (2017) A new personal mobility vehicle for daily life: improvements on a new RT-mover that enable greater mobility are showcased at the cybathlon. IEEE Robot Autom Mag 24(4):37-48

13. Takahashi M, Yoneda K, Hirose S (2006) Rough terrain locomotion of a legwheel hybrid quadruped robot. Proceedings of the 2006 IEEE international conference on robotics and automation, pp 1090-1095

14. Nakajima S (2017) Evaluation of the mobility performance of a personal mobility vehicle for steps. IEEE Access 5(1):9748-9756

\section{Publisher's Note}

Springer Nature remains neutral with regard to jurisdictional claims in published maps and institutional affiliations.

\section{Submit your manuscript to a SpringerOpen ${ }^{\circ}$ journal and benefit from:}

- Convenient online submission

- Rigorous peer review

- Open access: articles freely available online

- High visibility within the field

- Retaining the copyright to your article

Submit your next manuscript at springeropen.com 